\title{
The enhancement of the cellulolytic activity of cellobiohydrolase I and endoglucanase by the addition of cellulose binding domains derived from Trichoderma reesei
}

\author{
M.A. Lemos ${ }^{\text {a }} *$, J.A. Teixeira ${ }^{\text {a }}$, M.R.M. Domingues ${ }^{b}$, M. Mota ${ }^{a}$, F.M. Gama ${ }^{a}$ \\ a Departamento de Engenharia Biológica, Universidade do Minho, Campus de Gualtar, 4710-057 Braga, Portugal \\ ${ }^{\mathrm{b}}$ Departamento de Química, Universidade de Aveiro, Campus de Santiago, 3810-193 Aveiro, Portugal \\ Received 13 December 2001; received in revised form 16 August 2002; accepted 6 September 2002
}

\begin{abstract}
The effect of isolated cellulose binding domains (CBDs) on the hydrolysis of filter paper and microcrystalline cellulose by both cellobiohydrolase I and endoglucanase, was studied. CBDs were obtained by proteolysis from cellulases using a scaled-up variant of our previous method. Experiments were performed for different enzyme/substrate ratios in both the absence and presence of CBDs. Hydrolysis of filter paper by intact cellobiohydrolase I in the presence of additional CBDs was found to have a synergistic effect, leading to an increase of the sugar production of up to $30 \%$. The effect was less pronounced using microcrystalline cellulose, where an increase up to $16 \%$ was observed. Similar trends were found during the hydrolysis of both substrates by endoglucanase.
\end{abstract}

(C) 2002 Elsevier Science Inc. All rights reserved.

Keywords: Cellulose binding domains; Synergism; Cellulases

\section{Introduction}

Cellulases and hemicellulases have a wide range of application in biotechnology, such as in the food industry, pulp and paper industry, textile and laundry [1]. Cellulases are enzymes able to hydrolyse cellulose and the hydrolysis of crystalline cellulose can be performed by a fungal cellulase system, where three main enzymes play a pivotal role. These are (i) cellobiohydrolases $(\mathrm{CBH})$, which act as an exoglucanase releasing cellobiose as the main product, (ii) endoglucanases (EG) that act randomly along cellulose chains producing new sites for the cellobiohydrolases, and (iii) $\beta$-glucosidase, which hydrolyses cellobiose, a potential inhibitor of $\mathrm{CBH}$. This co-operative system involves the so-called "synergism effect," i.e. an enhancement of the activity over the added activities of the individual enzymes. The so-called exo-endo synergism has been described in the literature [2-5] and is normally explained by the fact that EG can provide free end chains on the cellulose surface for the $\mathrm{CBH}$ to act on. Furthermore, both exo-exo synergism [6-9] and endo-endo synergism can be observed [10].

\footnotetext{
${ }^{*}$ Corresponding author.
}

The structure of most cellulases consists of a catalytic domain (CD) connected by a glycosilated linker to a cellulose binding domain (CBD), which has an important role in the binding of these enzymes to cellulose. In order to evaluate the importance of the CBD in relation to endoglucanase E (CelE), from Pseudomonas fluorescens subsp. cellulose, Hall et al. [11], performed a study using the full-length enzyme CelE and a truncated form, lacking the CBD. Different cellulose substrates were hydrolysed and it was found that both forms exhibited similar activities against soluble forms of cellulose. However, CelE displayed approximately four times higher activity than the truncated form against Avicel. Do CBDs by themselves change the structure of the cellulose substrates? In fact, there are different data to be found in literature to answer this question. For instance, Din et al. [12] observed that the isolated CBD of endoglucanase A (CenA) from Cellulomonas fimi disrupted the structure of cellulose fibres and released small particles. On the other hand, Gill et al. [13] concluded that $\mathrm{CBD}_{\mathrm{X}}$ (CBD of $P$. xylanase A) did not disrupt the cell-wall material or bacterial microcrystaline cellulose and did not enhance the activity of the catalytic domain when not covalently linked to the enzyme. This confirmed the results obtained by Carrard et al. [14], which showed that, by covalently linking a CBD to an endoglucanase from $C$. thermocellum (CelD), 
an increase of the enzyme activity is observed. However, when free CBDs were added to the enzyme, the enzyme did not show this enhanced activity.

Taking into account the contradictions found in the literature related to the effect of CBDs on enzyme activity we decided to study the effect of additional CBDs on the hydrolysis of different substrates (using different enzyme/substrate ratios) by CBH I and EG. This was carried out using CBDs produced by a scaled-up adaptation of our previous method [15].

\section{Materials and methods}

\subsection{CBD production}

The production of CBDs was based on the previously published method [15], which was adapted to produce CBDs on a larger scale (in this work up to $600 \mathrm{mg}$ were produced with a yield of $76 \%$ ). A crude cellulase preparation (Celluclast, Novo Nordisk) was diluted $(4 \times)$ in sodium acetate buffer $(50 \mathrm{mM}, \mathrm{pH} 5)$ and "washed" in a hollow fibre cartridge (Amicon) with a $30 \mathrm{kDa}$ nominal weight cut-off polysulphone membrane to remove low molecular weight compounds along with any small endoglucanases. These could eventually become hydrolysed to give catalytic domains capable of passing through the hollow fibre cartridge $(10 \mathrm{kDa})$ used in the following step. Digestion was performed with papain (Sigma) using the ratio 300:1 (w/w) (cellulase/papain) at room temperature for $4 \mathrm{~h}$ with agitation. After digestion CBDs were obtained by ultrafiltration of the digestion mixture through a hollow fibre cartridge with a $10 \mathrm{kDa}$ nominal cut off. In order to detect and quantify any residual enzymatic activity, the filtrates were incubated for $1 \mathrm{~h}$ with carboximetilcellulose (CMC) $(1 \%)$ and filter paper (Whatman no. 1) at $50{ }^{\circ} \mathrm{C}$. The amount of sugar released was quantified by the dinitrosalicylic (DNS) acid method. The protein concentration in the cellulase preparation and filtrates was determined using the BCA Protein Assay Kit (Pierce). After testing for enzymatic activity and adsorption ability into cellulose, the filtrates were lyophilised and stored at $4{ }^{\circ} \mathrm{C}$.

\subsection{Peptide analysis}

This was accomplished using both capillary electrophoresis (CE) as previously described [15] and mass spectrometry. The latter was carried out using a MALDI-MS (Micromass, Manchester, UK), operating in linear mode, equipped with nitrogen laser with emission at $337 \mathrm{~nm}$. The matrix used was $\alpha$-cyano-4-hydroxycinnamic (Aldrich), which was recrystallised from methanol and dissolved in $\mathrm{H}_{2} \mathrm{O} /$ acetonitrile/trifluoroacetic acid $(50 / 50 / 0.1 \%)$ to form a saturated solution. An aqueous sample of the peptide was mixed with the matrix solution in equal proportions and then $1.5 \mu \mathrm{l}$ of this mixture was placed on the sample plate and

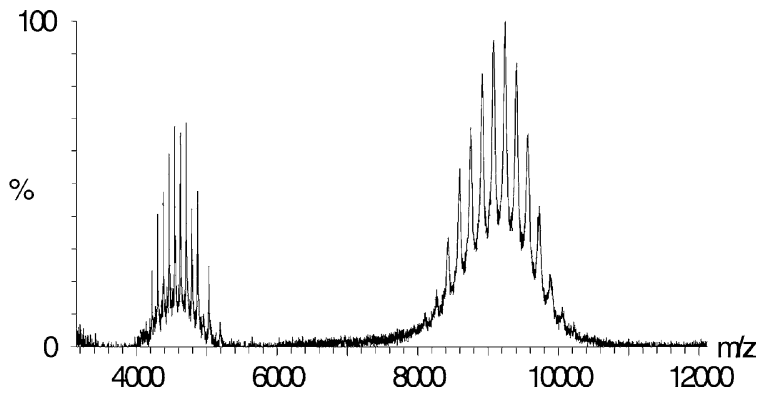

Fig. 1. Mass spectra of the peptide mixture.

allowed to dry. The results obtained are presented in Fig. 1 and show two distinct groups of peaks. Those observed in the higher mass region of the spectrum correspond to the molecular ions $[\mathrm{M}+2 \mathrm{H}]^{+}$of the peptides and exhibits an envelope centred around $9 \mathrm{kDa}$ (a value also obtained using CE). The multiple peaks observed are attributed to several degrees of glycosilation, since the difference between adjacent peaks corresponds to one hexose residue, which is unsurprising as papain acts in a non-specific manner on the glycosilated linker. The group of peaks observed in the lower mass region of the spectrum relate to the doubly charged ions $[\mathrm{M}+2 \mathrm{H}]^{2+}$. The molecular weight of the core binding domain of fungal cellulases is usually close to $5 \mathrm{kDa}$, however, if it remains heavily glycosylated a molecular weight of $10 \mathrm{kDa}$ can be expected [16]. Thus, it appears that glycosylated CBDs were obtained.

\subsection{Hydrolysis reactions}

Cellobiohydrolase I (crude enzyme preparation, Primalco biotec $\mathrm{H}$ ) and endoglucanase (crude enzyme preparation, Primalco biotec $\mathrm{G}$ ) were incubated at $50^{\circ} \mathrm{C}$ with filter paper (Whatman no. 1) and microcrystalline cellulose (Sigmacel, type 101) with the following different substrate/enzyme ratios: 5, 20, 25, 50, 200 and 250. Hydrolysis was performed for $48 \mathrm{~h}$ and at designated times samples were taken, centrifuged and the supernatant analysed for reducing sugars. The amount of sugar released was quantified by the dinitrosalicylic acid method. $\beta$-Glucosidase (Sigma) was added to the solution in order to avoid CBH I inhibition and to allow for a correct sugar determination. The same quantity of CBDs $(4 \mathrm{mg}$ ) was used in all experiments.

\subsection{Kinetic modelling}

Data analysis was performed using Microcal Origin Software and based on the kinetic model published by Nidetzky and Steiner [17]. For simplicity the data relating to the production of reducing sugar $(S)$ with time were fitted as the sum of two exponential components (Eq. (1)), as the initial modelling using one rate constant proved inadequate. The model employed takes into account the existence of two fractions in the substrate, whose relative proportions are represented 
by the pre-exponential components $a_{1}$ and $a_{2}$ (which have been normalised to unity). These substrate fractions differ in their capacity for enzyme adsorption and susceptibility to enzyme attack. In other words we have an initial fast process (rate constant $k_{1}$ ), where the "easy" substrate fraction is hydrolysed and a slow process (rate constant $k_{2}$ ), where the "difficult" substrate is hydrolysed. The data presented in Section 3 shows the difference between rate constants (for each enzyme/substrate ratio) to better elucidate any synergistic effects (if present):

$S=a_{1} \exp \left(-k_{1} t\right)+a_{2} \exp \left(-k_{2} t\right)$

\section{Results and discussion}

Modelling of hydrolysis data was done using Eq. (1) (described in Section 2). Figs. 2 and 3 represent the hydrolysis of filter paper and Sigmacel, respectively, showing the experimental data and their associated fitted functions. These figures demonstrate that the presence of additional isolated CBDs can affect the amount of reducing sugars released. It should be noted that when CBDs by themselves were incubated under similar conditions (as a control) with the two substrate materials no hydrolytic activity was detected. These data strongly indicate that the enhancement of sugar production can be attributed to the combination of enzyme and $\mathrm{CBD}$ and not to any residual activity in the CBD solution. CBDs, should not in fact display any hydrolytic activity, but the control experiment also helps to rule out any contaminants, that could exhibit activity.

In order to further understand these results the differences between rates obtained with and without CBDs were anal- ysed. Fig. 4 compares the kinetic constants $\left(k_{1}\right.$ and $\left.k_{2}\right)$ obtained for both CBH I and EG for different substrate/enzyme ratios with the two substrates and compares samples with CBDs relative to those without. From this figure, it easy to notice that there are differences according to the enzyme used. Related to hydrolysis of Sigmacel by endoglucanase, it seems that the rates $\left(k_{1}\right.$ and $\left.k_{2}\right)$ are not affected by the presence of CBDs, since $k_{\mathrm{CBD}} / k$ is in all the cases approximately 1 . Also in the hydrolysis of filter paper by endoglucanase it appears that the initial rate $\left(k_{1}\right)$ is not influenced by the presence of CBDs. Nevertheless, the $k_{2}$ rate is affected by the presence of CBDs. In fact the major difference occurs in the second "phase" of the reaction. This can be explained by the fact that initially the enzyme attacks the part of the substrate that is easily hydrolysed, without the need of any "help" from the CBD. However, at later times and since the "easy" substrate is already hydrolysed, the presence of the CBD plays an important role in the hydrolysis of filter paper by the endoglucanase.

When looking at the effect of CBDs on cellulose hydrolysis by $\mathrm{CBH}$, it is not so clear that the main role of the CBDs only occurs at this second phase of the reaction as overall there is a slight enhancement in the initial $\left(k_{1}\right)$ rate. A similar behaviour to EG using filter paper is seen in the $k_{2}$ rate constant and $\mathrm{CBH} I$ in the presence of isolated CBDs also produces an effect with Sigmacel as the substrate. It is well known that endoglucanases and exoglucanases act differently in the cellulose chains, which can explain also the difference in the rates observed for EG and CBH I. The enhancement of enzyme activity by the presence of additional isolated CBDs is also dependent on the hydrolysis mechanism of the enzyme. Related to the pre-exponential $a$ parameter, no difference was found for the celluloses "treated"

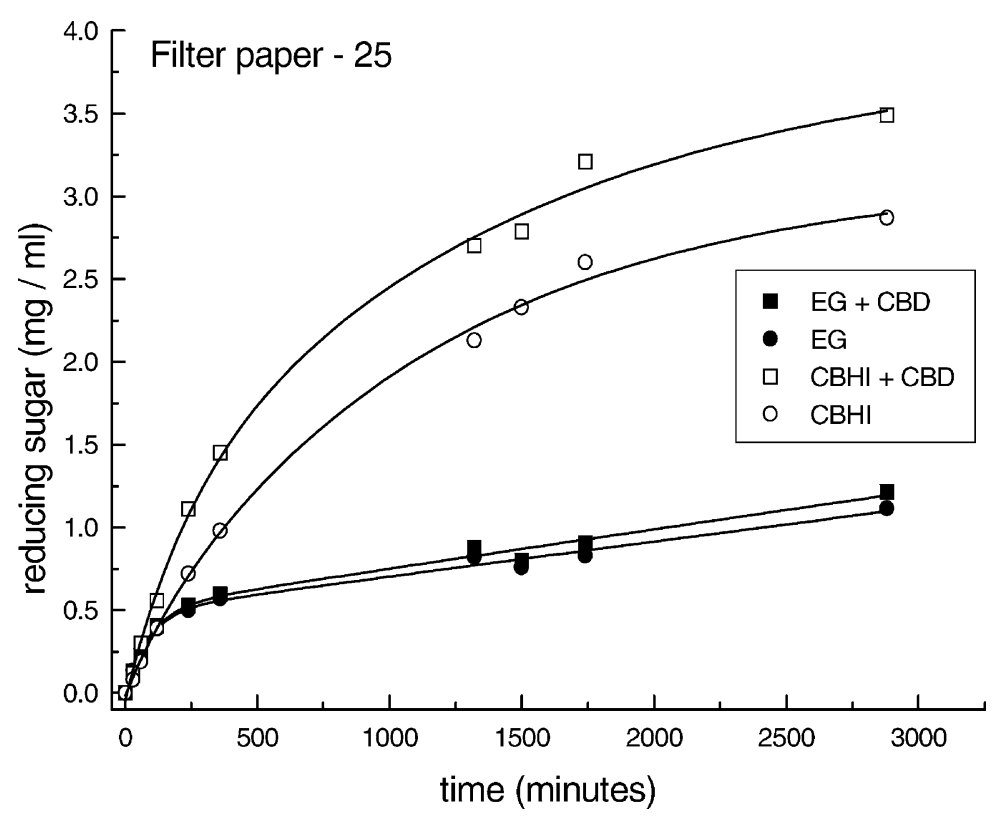

Fig. 2. Data and fit for the hydrolysis of filter paper by endoglucanase and cellobiohydrolase I (in the presence and absence of additional isolated CBDs) using the ratio 25 . 


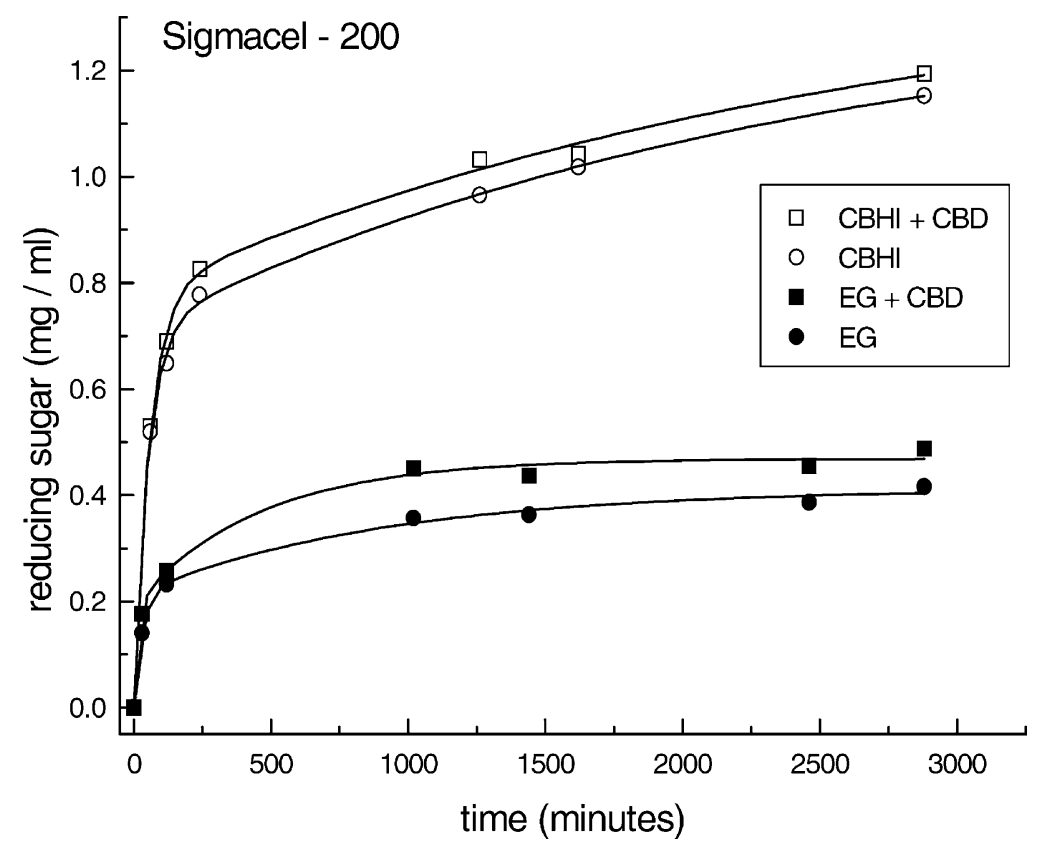

Fig. 3. Data and fit for the hydrolysis of Sigmacel by endoglucanase and cellobiohydrolase I (in the presence and absence of additional isolated CBDs) using the ratio 200 .
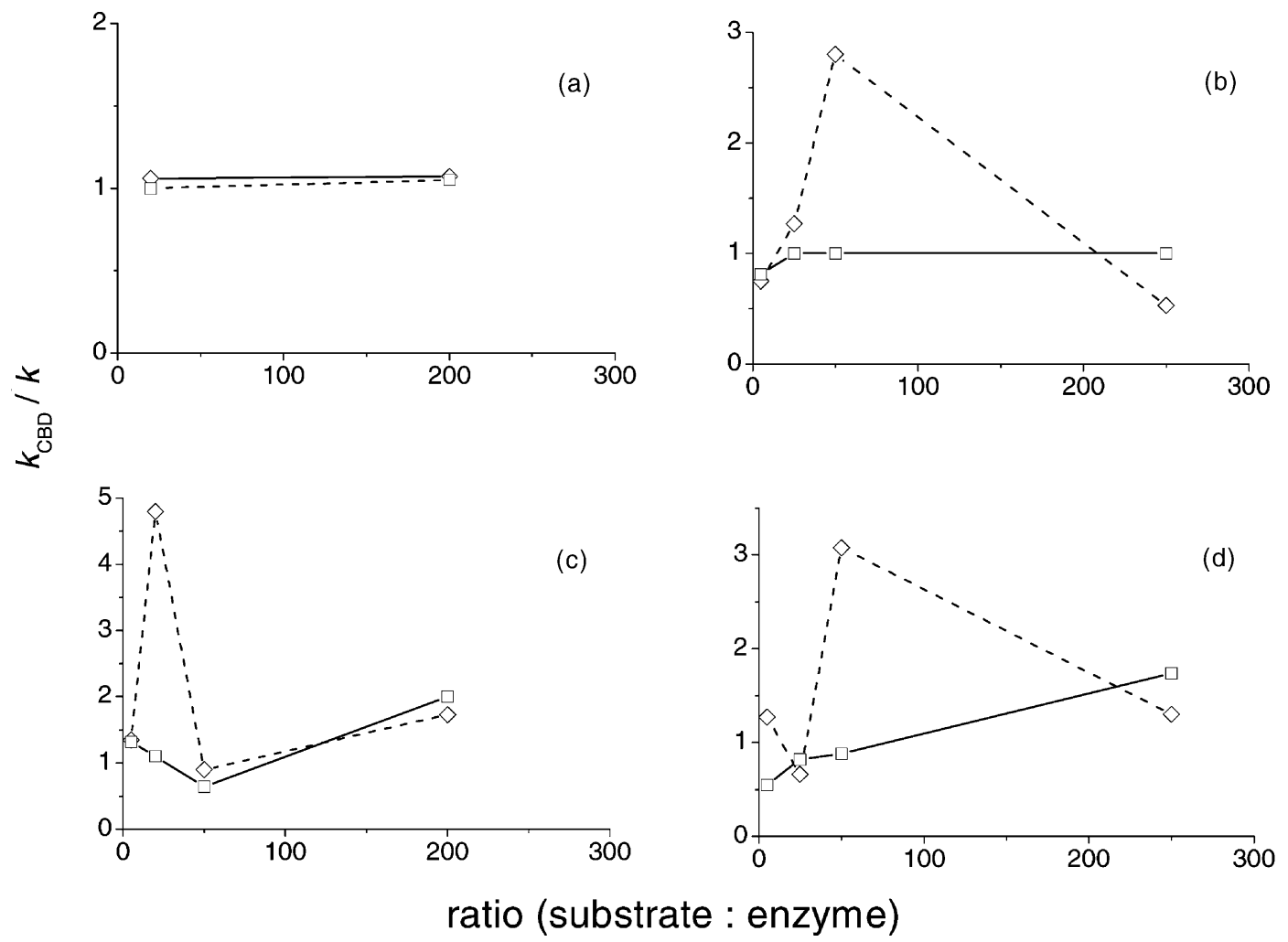

Fig. 4. Differences in the two rates $\left(\square, k_{1-\mathrm{CBD}} / k_{1}\right.$ and, $\diamond, k_{2}$-CBD $\left./ k_{2}\right)$ obtained with and without the presence of CBDs for the systems of: (a) endoglucanase, Sigmacel; (b) endoglucanase, filter paper; (c) cellobiohydrolase I, Sigmacel and (d) cellobiohydrolase I, filter paper. 


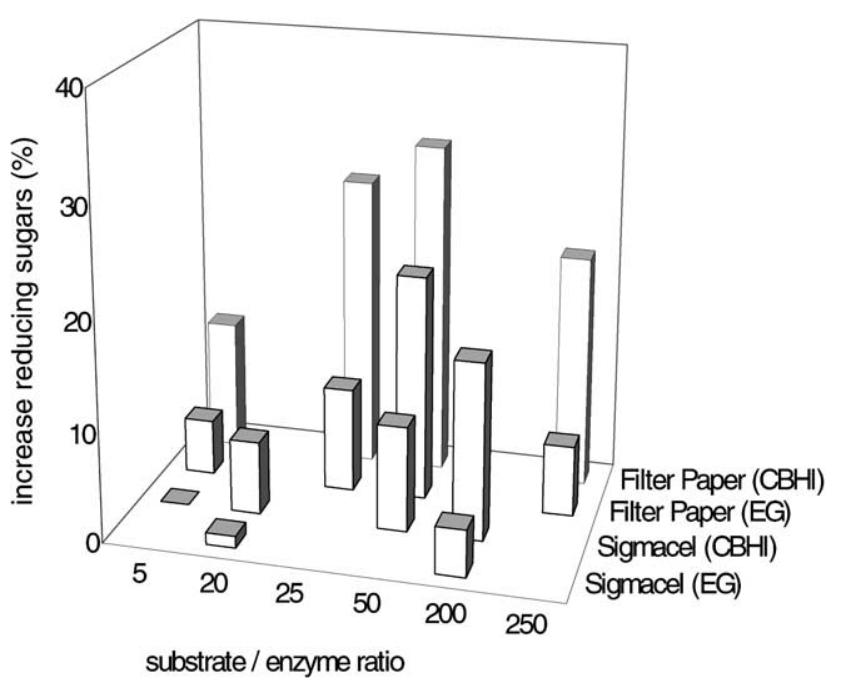

Fig. 5. Overall increase of sugar production by endoglucanase and cellobiohydrolase, comparing different substrate/enzyme ratios and type of substrate, when CBDs were added to the enzyme solution.

with CBDs. As shown by Nidetzky and Steiner [17], in fact $a$ does not seem to be related to physochemical properties of cellulose (such as crystallinity, degree of polymerisation).

Fig. 5 shows the overall increase of the production of reducing sugar, compared with the use of the single enzyme, for different substrate/enzyme ratios and for each type of substrate. In all cases reducing sugar production was found to be higher when using the combination of additional CBD and enzyme. Hydrolysis of filter paper by native cellobiohydrolase I in the presence of CBDs was found to lead to an increase of the sugar production of up to $30 \%$. The effect was also present, albeit to a lesser extent when using microcrystalline cellulose, where an increase of up to $16 \%$ was observed. However, the increase, when compared to enzyme alone assays varies depending on the ratio used.

The fact that substrate concentration (or substrate/enzyme ratio) influences the level of synergism observed may be explained in the following manner. At high substrate concentrations (high substrate/enzyme ratio) the enzyme rapidly interacts with the larger number of substrate sites easily available for hydrolysis, which can explain the decrease of synergistic effects. On the other hand, at low substrate concentrations (low substrate/enzyme ratio) the low synergetic effect can be explained because of the high saturation of the substrate. This was confirmed by Medve et al. [8], who in the case of cellulases, showed that high saturation leads to a low adsorption hinders the substrate hydrolysis and consequently, interferes with synergism. Probably the CBD-enzyme may also be affected by this process leading to the low synergistic effects observed. The dependence of the synergistic effects on the substrate/enzyme ratio can, in part, explain the different results found by Nidetzky et al. [9] and by Woodward et al. [18]. Nidetzky also found (as we did using CBDs and enzyme) that the synergistic action between cellulases was extremely dependent on the type of cellulose used and this effect was low on easily accessible celluloses.

Visual observation of the suspensions along the reaction time allowed the detection of a noticeable difference between the suspensions with and without CBDs. In the suspensions of microcrystalline cellulose (Sigmacel) without CBDs, the cellulose showed a tendency to agglomerate (precipitate) faster than the suspensions with CBDs. Similarly, the suspension of filter paper with enzyme and CBDs showed the presence of smaller particles compared with that without CBDs. The stabilising effects of adsorbed enzyme/protein on smaller particles in aqueous media has previously been observed [19]. CBDs without enzyme, however, did not change in any obvious manner the structure of filter paper. Several reports demonstrate that CBD can modify cellulosic fibres. Din et al. [12] showed that the isolated CBD from endoglucanase A (CenA) disrupted the structure of cellulose (ramie cotton fibres) releasing small particles. Also Pala et al. [20] showed that CBDs can have a positive effect on pulp characteristics (mainly pulp drainage rate and paper resistance indexes). This can probably be explained in terms of changes in the surface/interfacial properties of the fibres. In fact, how the CBDs can produce this enhancement of enzyme activity is not at present fully understood. However, it is possible to say that the effect of CBDs seems to be dependent on the fibre structure. This can also explain the differences found in this work when two different substrates were used-a more pronounced synergistic effect was detected with filter paper. Thus, the extent of synergism can be related to how easy a substrate can be hydrolysed by an enzyme and the CBD can be a helpful "tool" to enhance the enzyme hydrolysis of more complex fibre structures.

A recently published work [21] can assist in clarifying certain interactions of CBDs with celluloses, as researchers have shown that the CBD from EGIII, obtained by genetic engineering could change the morphology and structure of cellulose substrates. This leads to a decrease in both the crystallinity index and the number of hydrogen bonds. Another factor which can play an important role and requires further study is the timescale of incubation. In most works this is in the order of several hours, however, in this study by Xiao et al. [21] where they found changes in the structure of cellulose caused by the presence of CBDs, used an incubation time about 10 times longer. In our work an incubation time of $48 \mathrm{~h}$ was used and as in some cases the synergistic effect is more apparent in the $k_{2}$ rate controlled (longer timescale) process and therefore, care must be taken to ensure that no effect has been overlooked (or not detected).

\section{Conclusion}

In this study, we have shown that unlinked, isolated CBDs from Trichoderma reesei (obtained by proteolysis), when they are added to the solution produce an enhancement in the hydrolysis of cellulose (in both microcrystalline and filter 
paper) by intact CBH I and EG. The hydrolysis was found to be adequately modelled by a two stage process and the major synergism was found in the second phase, after "easy" substrate has been hydrolysed by enzyme with little or no assistance by the CBD. Also using filter paper produced higher levels of synergism hinting that the substrate structure and interaction with CBDs may be important.

Summarising the synergistic effect was found to be dependent of (i) enzyme, (ii) substrate/enzyme ratio (a maximum was found at 50 for both CBH I and EG), (iii) type of substrate and (iv) time of the hydrolysis. Taking into account the variety of factors that can affect the synergistic effect it is not surprising that we might find different data in literature. Moreover, the source of the CBDs used in the experiments is also important. However, the exact mechanism by which the synergistic effect is manifest is not yet clear and further work related to structural changes in celluloses induced by CBDs, using techniques such as scanning electron microscopy, X-ray diffraction and IR spectroscopy, could prove fruitful.

\section{Acknowledgments}

M.A. Lemos would like to thank Fundação para a Ciência e Tecnologia (Programa Praxis XXI) for financial support. Thanks also to Graham Hungerford (Departamento Física, Universidade Minho) for discussions and kinetic analysis. M. Rosário, M. Domingues would like to thank to Micromass (Manchester) for the operation on the MALDI-TOF MALDI mass spectrometer.

\section{References}

[1] Bhat MK. Cellulases and related enzymes in biotechnology. Biotechnol Adv 2000;18:355-83.

[2] Nidetzky B, Steiner W, Hayn M, Claeyssens M. Cellulose hydrolysis by the cellulases from Trichoderma reesei: a new model for synergistic interaction. Biochem J 1994;298:705-10.

[3] Irwin DC, Spezio M, Walker LP, Wilson DB. Activity studies of eight purified cellulases: specificity, synergism, and binding domain effects. Biotechnol Bioeng 1993;42:1002-13.

[4] Medve J, Karlsson J, Lee D, Tjerneld F. Hydrolysis of microcrystalline cellulose by cellobiohydrolase I and endoglucanase II from Trichoderma reesei: adsorption, sugar production pattern and synergism of the enzymes. Biotechnol Bioeng 1998;59:621-34.

[5] Srisodsuk M, Kleman-Leyer K, Keränen S, Kirk TK, Teeri TT. Modes of action on cotton and bacterial cellulose of homologous endoglucanase-exoglucanase pair from Trichoderma reesei. Eur J Biochem 1998;251:885-92.

[6] Kim DW, Jang YH, Jeong YK. Adsorption behavoirs of two cellobiohydrolases and their core proteins from Trichoderma reesei on Avicel PH 101. Biotechnol Lett 1997;19(9):893-7.

[7] Tomme P, Heriban V, Claeyssens M. Adsorption of two cellobiohydrolases from Trichoderma reesei to Avicel: evidence for "exo-exo" synergism and possible "loose-complex" formation. Biotechnol Lett 1990;12(7):525-30.

[8] Medve J, Stählberg J, Tjerneld F. Adsorption and synergism of cellobiohydrolase I and II of Trichoderma reesei during hydrolysis of microcrystalline cellulose. Biotechnol Bioeng 1994;44:1064-73.

[9] Nidetzky B, Hayn M, Macarron R, Steiner W. Synergism of Trichoderma reesei cellulases while degrading different celluloses. Biotechnol Lett 1993;15:71-6.

[10] Mansfield SD, Saddler JN, Gübitz GM. Characterization of endoglucanases from the brown rot fungi Gloeophyllum sepiarium and Gloeophyllum trabeum. Enzyme Microb Technol 1998;23:13340.

[11] Hall J, Black GW, Ferreira LMA, Millward-Sadler SJ, Ali BRS. The non-catalytic cellulose-binding domain of a novel cellulase from Pseudomonas fluorescens subsp. cellulosa is important for the efficient hydrolysis of Avicel. Biochem J 1995;309:749-56.

[12] Din N, Gilkes NR, Tekant B, Miller Jr RC, Anthony RJ. Non-hydrolytic disruption of cellulose fibres by the binding domain of a bacterial cellulase. Biotechnology 1991;9:1096-9.

[13] Gill J, Rixon JE, Bolam DN, Mcqueen-Mason S, Simpson PJ, Williamson MP, et al. The type II and X cellulose-binding domains of Pseudomonas xylanase A potentiate catalytic activity against complex substrates by a common mechanism. Biochem J 1999;342(2):473-80.

[14] Carrard G, Koivula A, Söderlund H, Béguin P. Cellulose-binding domains promote hydrolysis of different sites on crystalline cellulose. Proc Natl Acad Sci USA 2000;19:10342-7.

[15] Lemos MA, Teixeira JA, Mota M, Gama M. A simple method to separate cellulose-binding domains of fungal cellulases after digestion by a protease. Biotechnol Lett 2000;22(8):703-7.

[16] Tomme P, Van Tilbeurgh H, Pettersson G, Van Damme J, Vandekerckhove J, Knowles J, et al. Studies of the cellulytic system of Trichoderma reesei QM 9414. Analysis of domain function in two cellobiohydrolases by limited proteolysis. Eur J Biochem 1988;170:575-81.

[17] Nidetzky B, Steiner W. A new approach for modelling cellulasecellulose adsorption and the kinetics of the enzymatic hydrolysis of microcrystalline cellulose. Biotechnol Bioeng 1992;42:469-79.

[18] Woodward J, Hayes MK, Lee NL. Hydrolysis of cellulose by saturating and non-saturating concentrations of cellulose: implications for synergism. Biotechnology 1988;6:301-4.

[19] Gama FM, Carvalho MG, Figueiredo MM, Mota M. Comparative study of cellulose fragmentation by enzymes and ultrasounds. Enzyme Microb Technol 1997;20:12-7.

[20] Pala H, Lemos MA, Mota M, Gama FM. Enzymatic upgrade of fold paperboard containers. Enzyme Microb Technol 29:274-9.

[21] Xiao Z, Gao P, Qu Y, Wang T. Cellulose-binding domain of endoglucanase III from Trichoderma reesei disrupting the structure of cellulose. Biotechnol Lett 2001;23:711-5. 